\title{
Research on the arc and the water gate valve continuum topology optimization design
}

\author{
Yifei Cai, Jianping Guo \\ Yunnan Institute of Water Hydropower Engineering Investigation, Design and Research, Yunnan \\ 650000, China
}

Keywords: Curved water gate valves, continuum, stability.

\begin{abstract}
About 983 years, the method of the invention by raising or lowering the water level to transport vessels, i.e. spaced from the construction of two dams, let the hull into the two pools formed by the dam. 1795 United States appear first canal with locks - Little Falls Canal, which uses wooden gate. Around 1830, there have been first metal gate. Hydraulic steel gate generally set at Hydro inlet and outlet positions, such as by closing or opening the gate to control the buildings over the water orifice to control the downstream water level, adjust the flow, resulting in flood control, power generation, irrigation, navigation and other benefits, and maintaining security of hydraulic structures. Radial gate with no gate slot, the smaller opening and closing force, good hydraulic conditions, are widely used as a working gate run on all types of waterways. Years of practice showed that the vast majority of good operating performance radial gate has withstood the test design conditions. However, due to the elongated arm radial gate pass water pressure is very sensitive to the hydrodynamic pressure, radial gate used in China for decades; there have been a lot of accidents. Jiguang Zhang and Liu Christine tolerance analysis of the 1960s and 1970s, the construction of more than 10 low-head curved steel gate crashed instance, the curved steel gate triggered the accident in accordance with the characteristics and causes of damage are divided into five categories: quasi-periodic impact damage; top of the door and At the same time destroy the door at the end of overflow; turning failure destruction; local open discharge and manufacture and installation of accident damage. Therefore, the significance of this study lies: in the initial design phase of construction projects get feature works according to the force structure of the optimal topology, saving time and effort; further complete the form according to the optimal topology, size optimization, the most close to the real force of the three-dimensional curved steel gate design, safe, easy to operate, save material, cost control and other purposes.
\end{abstract}

\section{Introduction}

Hydraulic steel gate is an important part of hydraulic structures, known as water conservancy project in the "safety valve", which is related to the safe operation of the entire Hydro safe and effective operation. In recent years, with the development of hydropower development, continue to build high-head dam work is also increasing the size of the gate, at home and abroad have appeared in a number of large or very large gate. Increasing the size of the gate, the gate structure on the seismic performance of a higher demand. Typically, the gate is an integral part of the activity, partially buried and hoisting equipment. Many types of gates, gate door by the appearance of the shape of the leaves can be divided into flat gate, gate, miter gate, arched gates and so on. Which, due to radial gate hoist effort, less embedded parts, tank and other advantages of nowhere, has been widely used? Structural optimization theory is one of the effective ways to improve the design of the gate. Currently, the new multi-gate research work focused on the late check gates and shape optimization, the use of topology optimization theory of optimization studies the emergence of hydraulic steel gate. In this paper, based on the continuum topology optimization theory, combined with structural finite element analysis software, representing a new systematic method of designing curved steel gate. In this paper, examples are given of the design of the new novel emerged oblique arm radial gate of the main steps and results. Into account the radial gate span / depth ratio differences were given low-span oblique arm arc steel gate design and large-span arc steel gate design methods discussed. 


\section{Fluid-structure coupling gates Computing Research}

Arbitrary Varangian, the structure of the Euler movement has been described in the Lagrangian coordinate system, while the fluid is in the free deformation space, and the movement of the coordinate system will be described. Arbitrary Lagrangian - Benefits Euler Lagrange method also has a single Euler method and single, while overcoming the shortcomings of each of the two methods, the method can be solved when the interface node using different sports description coordinated manner when faced with problems. Fluid motion to satisfy mass conservation equations, the equation of conservation of momentum and energy conservation equations. Viscous coefficient $\mu$ (coefficient of kinetic viscosity) of the fluid, its basic equations for conservation of mass equation:

Momentum conservation equation:

$$
\begin{aligned}
& \frac{\partial \mathrm{p}}{\partial \mathrm{t}}+\operatorname{div}(p u)=0 \\
& \frac{\partial}{\partial \mathrm{t}}(\rho u)+\operatorname{div}(\rho u \otimes u-P)=\rho F
\end{aligned}
$$

Among them $P=\left\{p_{i j}\right\}, p_{i j}=-p \delta_{i j}+\left(\mu \frac{\partial u}{\partial \mathrm{u}_{\mathrm{j}}}+\frac{\partial u_{j}}{\partial \mathrm{u}_{\mathrm{j}}}-\frac{2}{3} \operatorname{divu} \delta_{i j}\right)+\mu^{\prime} \operatorname{divu} \delta_{i j}$

$\mu=\lambda+\frac{2}{3} \mu$, Called expansion coefficient of viscosity.

Energy conservation equation:

$$
\frac{\partial}{\partial \mathrm{t}}\left(\rho e+\frac{1}{2} \rho u^{2}\right)+\operatorname{div}\left[\left(\rho e+\frac{1}{2} \rho u^{2}\right) u-p u\right]=\operatorname{div}(\operatorname{kg} \operatorname{rad} T)+\rho F \bullet u
$$

Where $\mathrm{P}$ is the pressure $\rho$ is density; $\mathrm{T}$ is the absolute temperature; Fluid velocity $u=\left(u_{1}, u_{2}, u_{3}\right)$, $u_{1}, u_{2}, u_{3}$ denote velocity components $\mathrm{x}, \mathrm{y}, \mathrm{z}$ direction said the divergence div. If the area is too infinitesimal point $x=\left(x_{1}, x_{2}, x_{3}\right) \mathrm{d} \mathrm{S}$, the unit normal vector $\mathrm{n}$. The volume of fluid flowing through the $\mathrm{n}$ ds along direction $[t, t+d t]$ is in $u \bullet n d S d t$, the quality of the fluid is $\rho u \bullet n d S d t$, the fluid momentum is $\rho u(u \bullet n) S d t=\rho(u \otimes u) n d S d t$.

Among them,

$$
u \otimes u=\left(\begin{array}{ccc}
u_{1}^{2} & u_{1} u_{2} & u_{1} u_{3} \\
u_{2} u_{1} & u_{2}^{2} & u_{2} u_{3} \\
u_{3} u_{1} & u_{3} u_{2} & u_{3}^{2}
\end{array}\right)
$$

Fluid pressure differential element of area $\mathrm{d} \mathrm{S} \mathrm{n}$ by the positive side is ends (opposite to the pressure direction and the normal direction).

\section{Topology Optimization Design Continuum computing}

Topology optimization concept is given the role of boundary conditions and loads, find the structure of power transmission in the region within the limits prescribed design an optimal way. Generally simple statement called "digging holes", namely: Where can I find the holes in the structure, number and size of holes; in the layout of the hole, to meet the displacement and stress constraints structural requirements, and to external loads delivered to the office holder. Topology optimization is often an objective to achieve optimal performance of the structure, such as the smallest, lightest, based on a certain frequency range.

A two-dimensional C-clip, the axis of symmetry along the MN, shown in Figure 1, the long $\mathrm{OB}=$ $100 \mathrm{~mm}$, width $\mathrm{OA}=80 \mathrm{~mm}, \mathrm{BC}=30 \mathrm{~mm}, \mathrm{CF}=40 \mathrm{~mm}$, the diameter of the semicircular arc $\mathrm{EF}=$ $20 \mathrm{~mm}$. A point constraint DOF 3, M-point linear displacement of all constraints, N-point vertical displacement constraints; $\mathrm{D}$ by $\mathrm{Y}$ junction to concentrate force $\mathrm{F}=100 \mathrm{~N}$, node $\mathrm{C}$ by $\mathrm{Y}$ to focus $\mathrm{F}=$ $-100 \mathrm{~N}$. Elastic modulus of $200 \mathrm{GPa}$, Poisson's ratio of 0.3 . Now the unit is divided into rectangular units of $40 \times 36$. Target: Structure smallest; constraints: the node C, D displacement in the Y-axis 
direction are less than 0.07 . Figure 2 shows the distribution of the optimal material under the above conditions.

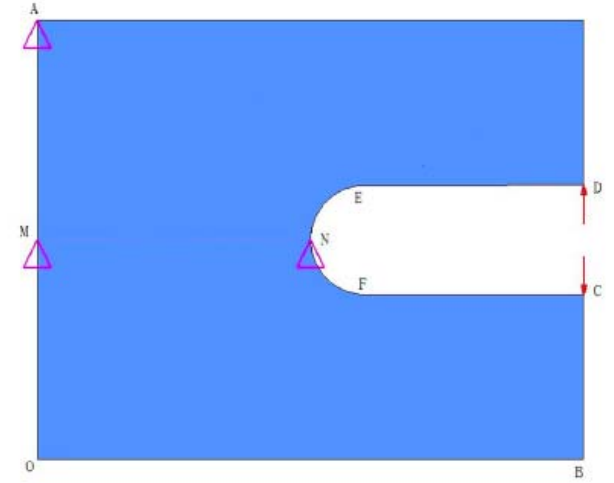

Fig. 1 Initial design domain

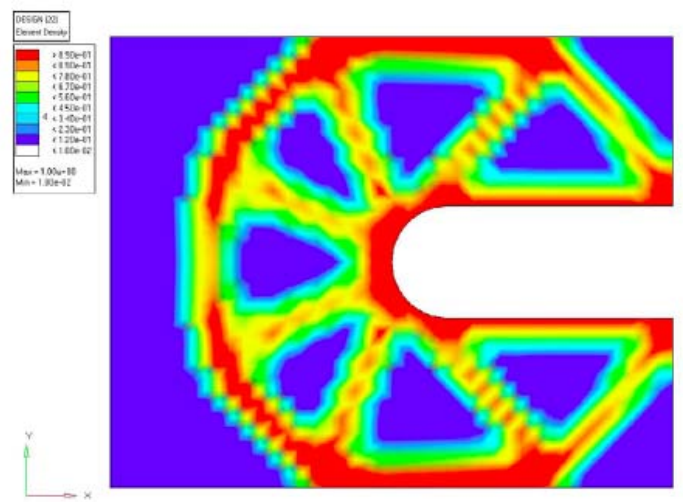

Fig. 2 The final topology of structure

Figure3: Two-dimensional structure length $1000 \mathrm{~mm}$, width $250 \mathrm{~mm}$. Non-design domains (black areas) by the upper uniform load $\mathrm{p}=100$, fixed at both ends of the bottom. Elastic modulus of $205 \mathrm{GPa}$, Poisson's ratio of 0.3 . Now the unit is divided into rectangular units of $25 \times 100$. Goal: Structure Flexibility minimum; constraints: the volume ratio of $20 \%$ remaining. Figure $2-4$ shows the distribution of the material under the above conditions.

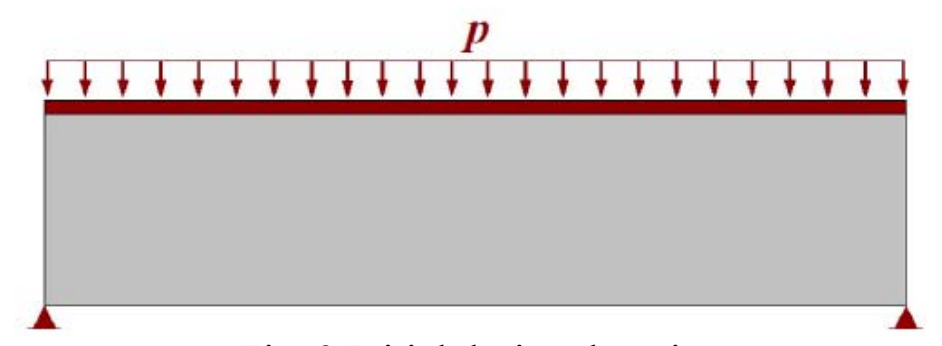

Fig. 3 Initial design domain

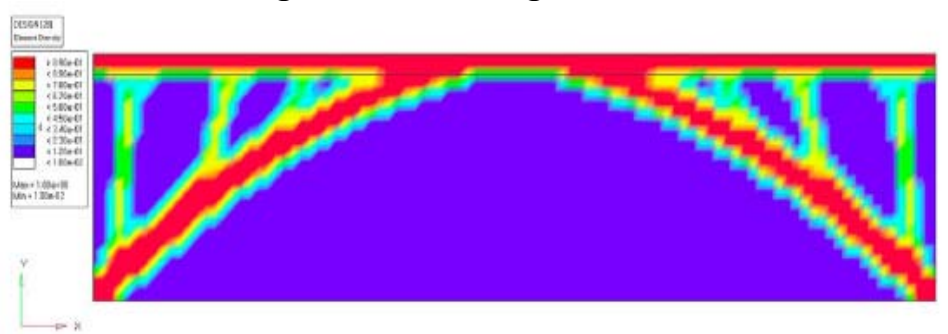

Fig. 4 The final topology of structure

\section{The resulting structure topology optimization optimal topology design residual material}

The shape of the lateral arm topology is known, is now based on known data, establish vertical arm design model is as follows: Arm material is still taken as Q345B steel, hydrostatic pressure q in accordance with the most adverse conditions, both full load conditions set in gear water upstream panel, the initial configuration of the design area in FIG. 5 (a) in Fig. Figure 5 (b) of the topological structure of the residual material after the optimal topology optimization to get. The following figure 5 (b) in a, b, c three o'clock position for the initial longitudinal three arms, a point along the straight line is rotated counterclockwise $2^{\circ}$. This method is applied to the topology optimization method hydraulic steel radial gate design first attempt to expand. The new structure meets the strength, stiffness and stability performance requirements. Demonstrate the feasibility of the continuum topology optimization theory in hydraulic steel radial gate design applications. 


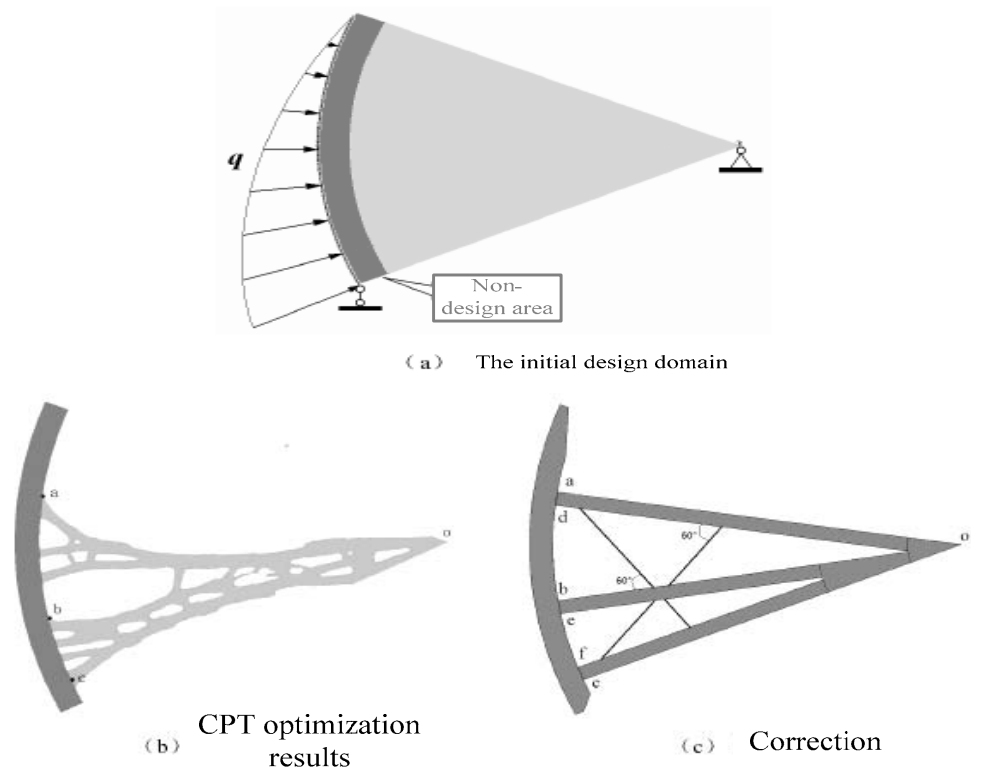

Fig. 5 Design of shape of a vertical arm

\section{Summary}

Currently, the new multi-gate research work focused on the late check gates and shape optimization. There is little research on how researchers use topology optimization theory to optimize arc steel gate. The topology optimization theory is applied to the gate design is very promising and meaningful work. I believe that with the development of computer technology in the future to tell, topology optimization theory and the finite element method to speak more complex problem solving. This article provides a preliminary design of a radial gate optimization, such as the idea of this paper gradually improve and optimize the design of hydraulic structures and innovations will provide more options, will promote the optimization of hydraulic structure design to achieve higher more economical design level. The above studies suggest that continuum topology optimization theory in hydraulic steel radial gate design is necessary, with significant engineering value.

\section{References}

[1]Das R, Jones R. Topology optimization of a bulkhead component used in aircrafts using an evolutionary algorithm. (2011)

[2]Guo Z, You S, Wan X, Bicanic N. 2010. A FEM-based direct method for material reconstruction inverse problem in soft tissue electrography. Computers \& Structures 88(23-24): p.1459 1468

[3]Jang I G, Kim I Y. 2010. Application of design space optimization to bone remodeling simulation of trabecular architecture in human proximal femur for higher computational efficiency.

[4]Lin P, Zhou P, Wu C W. 2011. Multi-objective topology optimization of end plates of proton exchange membrane fuel cell stacks. Journal of Power Sources 196(3): p.1222 1228

[5]Luo Z, Luo Q, Tong L, Gao W, C S. 2011. Shape morphing of laminated composite structures with photostrictive actuators via topology optimization methods. Composite Structures 93.

[6]Querin O M, Victoria M, Marti P. 2010. Topology optimization of truss-like continua with different material properties in tension and compression. Structural and Multidisciplinary Optimization. 